\title{
Is total arch replacement combined with stented elephant trunk implantation justified for patients with chronic Stanford type A aortic dissection?
}

\author{
Li-Zhong Sun, MD, ${ }^{\text {a }}$ Rui-Dong Qi, MD, ${ }^{\mathrm{b}} *$ Qian Chang, MD, ${ }^{\mathrm{a}}$ Jun-Ming Zhu, MD, ${ }^{\mathrm{a}}$ Yong-Min Liu, MD, ${ }^{\mathrm{a}}$ \\ Chun-Tao Yu, MD, ${ }^{a}$ Bin Lv, MD, ${ }^{\mathrm{c}}$ Jun Zheng, MD, ${ }^{\mathrm{a}}$ Liang-Xin Tian, MD, ${ }^{\mathrm{a}}$ and Jin-Guo Lu, MD
}

\begin{abstract}
Objective: Surgical treatment of chronic Stanford type A aortic dissection using total arch replacement combined with stented elephant trunk implantation is controversial owing to the visceral arteries and intercostal arteries originating from the false lumen.
\end{abstract}

\begin{abstract}
Methods: Eighty-nine patients (mean age, $45.67 \pm 10.18$ years; range, 21-68 years) with chronic type A dissection underwent total arch replacement combined with stented elephant trunk implantation between April 2003 and March 2007. Careful assessment of the visceral arteries and location of entry and re-entry was done before surgery. Postoperative patency of the visceral arteries and diameter of the aortic artery and the residual false lumen were evaluated by computed tomography.

Results: One $(1.12 \%)$ hospital death and $2(2.25 \%)$ late deaths occurred at a mean follow-up of 28.5 months (range, 8-52 months). Visceral malperfusion was not observed. Two patients had spinal cord injury and recovered during follow-up. One patient had a transient neurologic deficit and recovered completely before discharge. One patient underwent thoracoabdominal aortic replacement for aneurysmal dilatation of the residual descending aorta 3 months after the operation. Thrombus obliteration of the false lumen at the distal edge of the stented elephant trunk and at the diaphragmatic level was $94.2 \%(81 / 86)$ and $61.6 \%(53 / 86)$, respectively.
\end{abstract}

Conclusions: Satisfactory results with low morbidity and mortality were obtained. No visceral malperfusion and a low risk of postoperative spinal cord injury favor this technique in patients with chronic type A dissection.

Chronic type A dissection is not uncommon in China owing to (1) aortic dissection after previous cardiovascular surgery, ${ }^{1}(2)$ dilatation of the aorta distal to the aortic graft after successful surgical treatment of acute type A dissection using only ascending aortic replacement, ${ }^{2}$ and (3) delayed diagnosis and surgical treatment in patients with acute type A dissection. Surgical management of chronic type A dissection remains a formidable challenge, ${ }^{3,4}$ and several procedures have been developed. ${ }^{5-7}$

Total replacement of the arch combined with stented elephant trunk implantation had been proven to be feasible in patients with type A dissection in preliminary studies. ${ }^{8}$ Surgical treatment of chronic type A dissection using this technique is controversial owing to the visceral arteries and

\footnotetext{
From the Department of Cardiovascular Surgery, Cardiovascular Institute and Fuwai Hospital, Peking Union Medical College, Chinese Academy of Medical Sciences, ${ }^{a}$ Beijing, the Department of Cardiovascular Surgery, Tianjin Cardiovascular Institute and Tianjin Chest Hospital, ${ }^{\mathrm{b}}$ Tianjin, and the Department of Radiology, Cardiovascular Institute and Fuwai Hospital, Peking Union Medical College, Chinese Academy of Medical Sciences, ${ }^{\mathrm{c}}$ Beijing, China.

*Li-Zhong Sun and Rui-Dong Qi contributed equally to this work.

Received for publication Oct 14, 2008; revisions received Feb 9, 2009; accepted for publication Feb 22, 2009; available ahead of print April 27, 2009.

Address for reprints: Li-Zhong Sun, MD, Department of Cardiovascular Surgery, Cardiovascular Institute and Fuwai Hospital, 167 Beilishi Rd, Beijing, 100037, China (E-mail: slzh_2005@yahoo.com.cn).

J Thorac Cardiovasc Surg 2009;138:892-6

$0022-5223 / \$ 36.00$

Copyright (c) 2009 by The American Association for Thoracic Surgery doi:10.1016/j.jtcvs.2009.02.041
}

intercostal arteries originating from the false lumen. We reviewed our experience with the management of chronic type A dissection using this technique via a median sternotomy with the patients under hypothermic cardiopulmonary bypass (CPB) with selective cerebral perfusion (SCP).

\section{PATIENTS AND METHODS \\ Patient Data}

Eighty-nine patients ( 74 men and 15 women; mean age, $45.67 \pm 10.18$ years; range, 21-68 years) with chronic type A dissection underwent total arch replacement combined with stented elephant trunk implantation via a median sternotomy under hypothermic CPB with SCP from April 2003 to March 2007. This technique was approved by the Institutional Review Board of the Chinese Academy of Medical Science and Peking Union Medical College, Beijing, China. Informed consent was obtained from each patient. A history of hypertension was the commonest preoperative finding (Table 1).

The tear site was located at the ascending aorta in 36 patients, at the transverse arch in 17 patients, and at the proximal descending thoracic aorta in 26 patients. Among the remaining 10 patients, an entry tear was not found in 2 patients and previous surgery had been carried out in 8 patients (Bentall procedure for type A dissection in 3 patients; Bentall procedure for true aneurysms in 2 patients; ascending aortic replacement for type A dissection in 2 patients; and aortic valve replacement in 1 patient). The dissection extended into the distal descending thoracic aorta in 4 patients and into the abdominal aorta in 85 patients.

Preoperative computed tomography (CT) was done to carefully assess the visceral arteries. Table 2 shows the visceral arteries originating from the true lumen and false lumen. If visceral arteries originated from the a false lumen, re-entry was around the origin of visceral artery, as demonstrated by CT. Postoperative patency of the visceral arteries, diameter of the aortic artery, and the residual false lumen were evaluated by CT during follow-up. 


\section{Abbreviations and Acronyms \\ $\mathrm{CPB}=$ cardiopulmonary bypass \\ $\mathrm{CT}=$ computed tomography \\ $\mathrm{SCP}=$ selective cerebral perfusion}

\section{Stent Graft}

The stent graft (MicroPort Medical Company Limited, Shanghai, China $)^{9}$ was $10 \mathrm{~cm}$ long. The proximal and distal ends of the surgical stent graft had $1 \mathrm{~cm}$ of extra vascular graft, which was used for sewing. The anastomosis between the distal end of stented graft and a Dacron prosthesis was safer and easier if late thoracoabdominal aortic replacement was required. In patients with chronic type A dissection, appropriate sizing of the stented elephant trunk was close to the diameter of the proximal descending aorta of normal subjects matched for age, sex, and height. The diameter of the stent graft was larger than that of the true lumen and was smaller than that of the entire aorta as determined by preoperative $\mathrm{CT}$ and intraoperative evaluation.

\section{Surgical Technique}

The surgical technique has been described in detail by our research group. ${ }^{8,9}$ Total replacement of the aorta combined with stented elephant trunk implantation was done in all patients via a median sternotomy under $\mathrm{CPB}$ with SCP. Cannulation of the right axillary artery was routinely carried out for $\mathrm{CPB}$ and SCP. CPB and cooling were started after cannulation of the right axillary artery and the right atrium. Brachiocephalic arteries were dissociated from surrounding tissue during the cooling period and exposed. Aortic root procedures were done (if necessary) after ascending aortic crossclamping and cardioplegia. Circulatory arrest was established when the nasopharyngeal temperature reached $18^{\circ} \mathrm{C}$ to $22^{\circ} \mathrm{C}$. SCP was started through the right axillary artery, and the brain was perfused at approximately 5 to 10 $\mathrm{mL} \cdot \mathrm{kg}^{-1} \cdot \mathrm{min}^{-1}$. The ascending aorta and aortic arch were opened. The tear site was resected whenever possible. The proximal descending aorta was transected circumferentially distal to the origin of the left subclavian artery or distal to the intimal tear in the proximal descending aorta. Owing to recurrent laryngeal nerve injury, we altered the site of the distal anastomosis. The distal aorta was transected circumferentially close to the proximal margin of the origin of the left subclavian artery (the primary intimal tear in the proximal descending aorta was sealed off with the stented elephant trunk concomitantly). A $10-\mathrm{cm}$ long stent graft in a bound, compressed state was inserted into the true lumen of the descending thoracic aorta. After implantation in the correct location, the anastomosis between the fourbranched prosthetic graft and the descending aorta was done by the open aortic technique. Antegrade systemic perfusion was re-established via the perfusion limb of the prosthetic graft. The left common carotid artery was

TABLE 1. Characteristics of patients with chronic type A dissection

\begin{tabular}{lc}
\hline \multicolumn{1}{c}{ Variable } & No. \\
\hline Age (y) & $45.67 \pm 10.18$ \\
Male & 74 \\
Female & 15 \\
Hypertension & 56 \\
Diabetes mellitus & 5 \\
Marfan syndrome & 14 \\
Coronary disease & 8 \\
Lower extremity ischemia & 3 \\
Chronic renal dysfunction & 2 \\
Cerebrovascular disease & 2 \\
Acute left heart failure & 1 \\
Lower extremity ischemia & 3 \\
\hline
\end{tabular}

TABLE 2. Visceral arteries: from the true lumen or the false lumen?

\begin{tabular}{lccr}
\hline & False & True & Both \\
\hline Celiac artery & 13 & 54 & 22 \\
Superior mesenteric artery & 4 & 72 & 13 \\
Right renal artery & 24 & 60 & 5 \\
Left renal artery & 23 & 53 & 13 \\
\hline
\end{tabular}

then reconstructed. After the anastomosis was completed, CPB was gradually returned to normal flow, $\mathrm{SCP}$ was discontinued, and rewarming was started. Reconstruction of the left subclavian artery, the innominate artery, and the proximal anastomosis were accomplished.

\section{RESULTS}

\section{Surgical Data}

Patients with chronic type A dissection underwent total arch replacement combined with stented elephant trunk implantation under hypothermic CPB with SCP. Concomitant procedures are summarized in Table 3. CPB time was 100 to 315 minutes (mean, $179 \pm 39$ minutes); aortic crossclamp time was 50 to 190 minutes (mean, $105 \pm 28$ minutes); and the selective cerebral perfusion time was 9 to 48 minutes (mean, $25 \pm 7$ minutes).

\section{Mortality and Morbidity}

There was one $(1.12 \%)$ hospital death. The patient had a new dissection in the abdominal aorta after the operation, and visceral organ ischemia was observed. The patient then received thoracoabdominal aortic replacement but died of multiple-organ failure.

Injury to the spinal cord occurred in 2 patients. One had weakness in the left lower extremity, and the other patient had paraparesis; both recovered during the follow-up period. One patient had a transient neurologic deficit, and cerebral embolism was observed in 1 patient; both recovered completely before hospital discharge. A return to the operating room owing to bleeding was seen in 6 patients, hypotension of the lower extremities in 1 patient, thrombophlebitis in 1 patient, and recurrent laryngeal nerve injury in 1 patient.

\section{Imaging}

Patients underwent CT during follow-up. Thrombus obliteration of the false lumen at the distal edge of the stented

TABLE 3. Concomitant procedures

\begin{tabular}{lr}
\hline \multicolumn{1}{c}{ Variables } & No. \\
\hline Bentall procedure & 20 \\
Cabrol procedure & 4 \\
Wheat procedure & 1 \\
David procedure & 1 \\
Sinus of Valsalva reconstruction & 3 \\
Aortic valve repair & 7 \\
Aortic valve replacement & 4 \\
Coronary artery bypass graft & 5 \\
Ascending aorta-femoral artery bypass & 1 \\
Ascending abdominal bypass & 1 \\
\hline
\end{tabular}



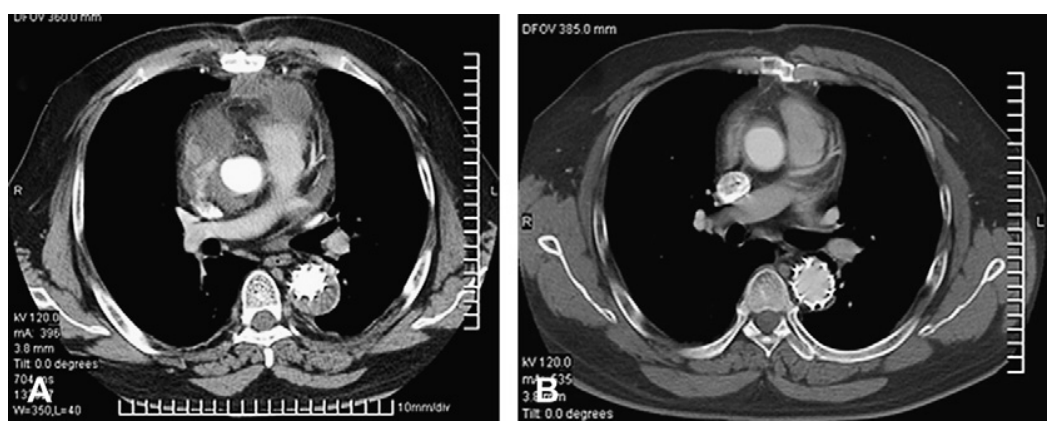

FIGURE 1. CT of a patient with chronic type A dissection 2 weeks (A) and 12 months (B) after the operation. The stented elephant trunk was compressed by the dissected membrane, and the false lumen was obliterated with thrombus (A). After remodeling of the aortic wall and thrombus reabsorption, the two dissected layers were successfully approximated to obliterate the false lumen by the stented graft (B).

elephant trunk was observed in $82(95.3 \%)$ patients (Figure 1). Persistent false lumen around the stented graft was observed in $4(4.7 \%)$ of 86 patients. Compared with preoperative data, the diameter of the descending aorta was $28.18 \pm 2.03 \mathrm{~mm}$ (vs $38.19 \pm 4.86 \mathrm{~mm})$ in patients with obliteration of the false lumen. In 4 patients with a patent false lumen, the diameter of the descending aorta was larger postoperatively than preoperatively $(41.89 \pm 5.07 \mathrm{~mm}$ vs $40.74 \pm 5.36 \mathrm{~mm}$ ). Disappearance or near complete disappearance of the false lumen in the descending aorta was observed in $49(55.1 \%)$ of 89 patients. The descending aorta returned to normal size after remodeling of the aortic wall. Complete formation of a thrombus at the level of the diaphragm was observed in $53(61.6 \%$ ) of 86 patients (Figure 2). Thrombus obliteration of the false lumen, absorption of false channel thrombosis, enlargement of the true lumen, and shrinkage in the diameter of the entire aorta developed in a continuous dynamic process during follow-up.

\section{Follow-up}

There were $2(2.25 \%)$ late deaths at a mean follow-up of $28.53 \pm 12.70$ months (range, $8-52$ months) and 2 of 86 were lost to follow-up. The 2 patients died of cerebral hemorrhage 14 and 16 months after the operation. Cerebral infarction occurred in 1 patient 31 months after the operation, but he recovered. Dilatation of the residual descending aorta was observed in 1 patient 3 months after the operation, and thoracoabdominal aortic replacement was done; the patient recovered after hospital discharge. Thrombosis of the celiac artery originating from the true lumen and the false lumen occurred in 1 patient before the operation. Fortunately, symptoms of visceral organ ischemia were not observed during follow-up. Injury to the spinal cord and visceral malperfusion were not observed after the operation.

\section{DISCUSSION}

Surgical management of chronic type A dissection remains a formidable problem when the ascending aorta, the aortic arch, and part (or all) of the descending aorta are involved. Several surgical procedures have been developed, ${ }^{5-7}$ but the optimal technique for the treatment of chronic type A dissection is not clearly established.

The conventional elephant trunk technique has been used most widely for staged aortic replacement in patients with chronic type A dissection, ${ }^{5}$ but several issues regarding this technique have been raised. It is virtually impossible to place the conventional elephant trunk in the true lumen because of compression of the rigid chronic dissecting membrane owing to the fibrotic intimal membrane.$^{10}$ Complications such as kinking and obstruction of the graft, embolization, and paraplegia have been observed. ${ }^{5}$ Deaths owing to rupture of the remaining aneurysmal aorta during the interval between the first and second stages of the procedure were seen. ${ }^{11}$ Selecting an appropriated interval between the two procedures was problematic. ${ }^{12}$ Some patients failed to return for the second-stage procedure. ${ }^{11}$

Kouchoukos and associates ${ }^{7}$ introduced the "arch-first" technique to treat chronic type A dissection via a bilateral anterior thoracotomy incision. They thought that the heart, the brachiocephalic arteries, the left phrenic and left vagus nerves, and the entire descending aorta were very well exposed. This benefited carrying out an anastomosis between the prosthetic graft and the vessels, carrying out the concomitant procedure, minimizing the duration of brain ischemia, and avoiding injury to adjacent organs, but both internal thoracic arteries were sacrificed and a high prevalence of pulmonary complications was observed. ${ }^{13}$

Some modifications of the stented elephant trunk procedure were made by our research group ${ }^{8}$ (we called it "Sun's procedure"). Preliminary results in patients with type A dissection using total arch replacement combined with stented elephant trunk implantation were encouraging. ${ }^{8}$ The stented elephant trunk technique has several advantages over the techniques discussed previously. In comparison with the conventional elephant trunk technique, applying the stented elephant trunk (which is in a bound, compressed state) into the descending aorta was very easy. Meanwhile, 

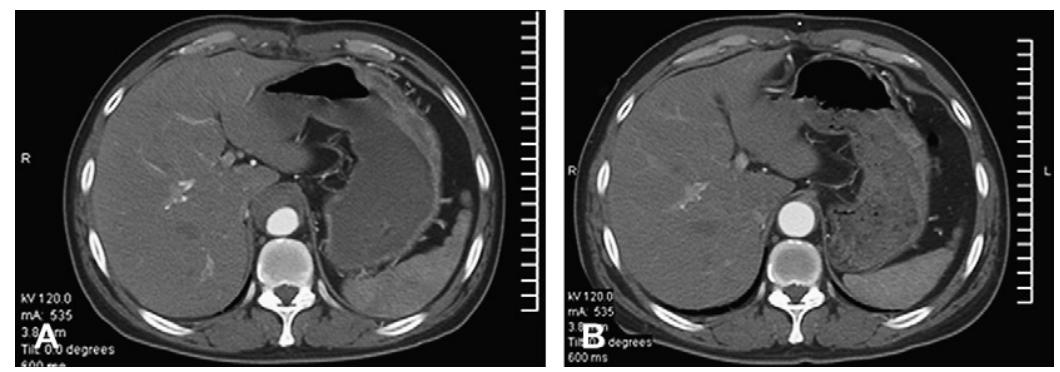

FIGURE 2. CT of a patient with chronic type A dissection 2 weeks (A) and 6 months (B) after the operation. The false lumen was obliterated with thrombus at the diaphragmatic level (A). The true lumen was resumed completely with absorption of false channel thrombosis, and the descending aorta returned to normal size after remodeling of the aortic wall (B).

the false lumen was obliterated with thrombus after the stented elephant trunk was implanted. In this group, thrombus obliteration of the false lumen in the descending aorta at the distal edge of the stented elephant trunk and at the diaphragmatic level was $95.3 \%(82 / 86)$ and $61.6 \%(53 / 86)$, respectively. The second operation may be avoided in most cases. Only one patient underwent thoracoabdominal aortic replacement for aneurysmal dilatation of the residual descending aorta 3 months after the operation. Aortic rupture in the interval between the first and second procedures was therefore avoided. This procedure would make it safer and easier to carry out the anastomosis between the distal end of the stent graft and the Dacron prosthesis if late thoracoabdominal aortic replacement was required. In contrast to the technique described by Kouchoukos and associates, sacrifice of the internal thoracic arteries was avoided and pulmonary complications were significantly reduced. Proximal descending aortic replacement could also be completed through a median sternotomy. ${ }^{14}$

The indications for this procedure for chronic type A dissection with dissection extending to the descending aorta or the abdominal aorta were as follows: (1) location of the intimal tear in the aortic arch or the proximal descending aorta (retrograde dissection); (2) involvement of the arch vessels; (3) aneurysmal dilatation of the aortic arch or descending thoracic aorta; and (4) Marfan disease. The stented elephant trunk could not work well while the diameter of the descending aorta was greater than $50 \mathrm{~mm}$. We recommend a staged total aortic replacement using this technique or a 1-stage total aortic replacement if the descending aorta is greater than $50 \mathrm{~mm}$ in diameter. ${ }^{6}$

The stented elephant trunk technique for chronic type A dissection remains controversial and centers on the visceral arteries and intercostal arteries supplied by the false lumen. Injury to the spinal cord, organ malperfusion, and chronic dissection of the membrane were confronted, and this technique was contraindicated in patients with chronic type A dissection.

Injury to the spinal cord is one of the most serious complications in patients with type A dissection using the stented elephant trunk technique, but the cause of paraplegia is not clear. This serous complication could be caused by spinal cord ischemia during lower body arrest, perioperative hypotension, embolism to intercostal arteries, and occlusion of critical intercostal arteries by the stented graft. A high prevalence of injury to the spinal cord has been reported, ${ }^{15}$ but the prevalence in our group was very low. In our opinion, ischemia in the chronic phase of chronic type A dissection enhances spinal cord tolerance to subsequent surgical ischemia by collateral circulation in patients with intercostal arteries from the false lumen. We think that injury to the spinal cord may be primarily related to lower body arrest, sacrifice of intercostal arteries that do not directly supply spinal cord blood flow but augment collateral blood flow, ${ }^{16}$ or several factors in combination in our group. To prevent occlusion of critical intercostal arteries below the Th8 level by the stented graft, we made the stented graft only $10 \mathrm{~cm}$ long and the distal end of the stented graft was above the Th8 level (as demonstrated by postoperative chest radiography or CT).

Organ malperfusion was another serious complication. A thrombosed false lumen would carry a risk of obstruction of the major branches supplied by the false lumen, but organ malperfusion was not observed in our group. We think that re-entry must be around the origin of the visceral artery when the visceral artery originated from the false lumen. Blood flow via the re-entry was supplied to visceral organs from the false lumen. When the false lumen was obliterated with thrombus, the distance between origin of the visceral artery and the intimal tear was shortened. Organ malperfusion would therefore be improved after thrombosis of the false lumen. Collateral arteries are also present between the celiac artery and the superior mesenteric artery. The organs, which are supplied by the celiac artery, were fed by collateral arteries from the superior mesenteric artery when the celiac artery was obliterated, and vice versa.

Another concern is whether the self-expanding stent graft can oppose the fibrous, potentially rigid, chronically dissected membrane against the native aortic wall. After the stented elephant trunk is implanted, the stented graft is substantially compressed by the rigid chronically dissected membrane. In our study, it may take several months (or even longer) to fully expand the stented graft. The two 
chronically dissected aortic layers can be successfully approximated to obliterate the false lumen using a stented elephant trunk. Persistent false lumen around the stented graft was observed in only 4 patients in this group.

However, this study documented our limited experience with a mean follow-up of $28.53 \pm 12.70$ months (range, 8-52 months). Data were preliminary and longer followup is required to validate the effectiveness of this technique for chronic type A dissection.

\section{CONCLUSION}

Satisfactory results with low morbidity and mortality were obtained in patients with chronic type A dissection using the stented elephant trunk technique. No visceral malperfusion and a low risk of injury to the spinal cord were observed in these patients. The encouraging results of the present study strengthen the case for clinical application of the stented elephant trunk technique in patients with chronic type A dissection.

\section{References}

1. Hirose H, Svensson LG, Lytle BW, Blackstone EH, Rajeswaran J, Cosgrove DM. Aortic dissection after previous cardiovascular surgery. Ann Thorac Surg. 2004; 78:2099-105.

2. Heinemann M, Laas J, Karck M, Borst HG. Thoracic aortic aneurysms after acute type A aortic dissection: necessity for follow-up. Ann Thorac Surg. 1990;49:580-4.

3. Fann JI, Smith JA, Miller DC, Mitchell RS, Moore KA, Grunkemeier G, et al. Surgical management of aortic dissection during a 30-year period. Circulation. 1995; 92(9 Suppl):II113-21.

4. Svensson LG, Crawford ES, Hess KR, Coselli JS, Safi HJ. Dissection of the aorta and dissecting aortic aneurysms. Improving early and long-term surgical results. Circulation. 1990;82(5 Suppl):IV24-38.
5. Crawford ES, Coselli JS, Svensson LG, Safi HJ, Hess KR. Diffuse aneurysmal disease (chronic aortic dissection, Marfan, and mega aorta syndromes) and multiple aneurysm. Treatment by subtotal and total aortic replacement emphasizing the elephant trunk operation. Ann Surg. 1990;211:521-37.

6. Hu XP, Chang Q, Zhu JM, Yu CT, Liu ZG, Sun LZ. One-stage total or subtotal aortic replacement. Ann Thorac Surg. 2006;82:542-6.

7. Kouchoukos NT, Masetti P, Rokkas CK, Murphy SF. Single-stage reoperative repair of chronic type A aortic dissection by means of the arch-first technique. J Thorac Cardiovasc Surg. 2001;122:578-82.

8. Liu ZG, Sun LZ, Chang Q, Zhu JM, Dong C, Yu CT, et al. Should the "elephant trunk" be skeletonized? Total arch replacement combined with stented elephant trunk implantation for Stanford type A aortic dissection. J Thorac Cardiovasc Surg. 2006;131:107-13

9. Sun LZ, Qi RD, Chang Q, Zhu JM, Liu YM, Yu CT, et al. Surgery for Marfan patients with acute type A dissection using a stented elephant trunk procedure. Ann Thorac Surg. 2008;86:1821-6.

10. Kieffer E, Koskas F, Godet G, Bertrand M, Bahnini A, Benhamou AC, et al. Treatment of aortic arch dissection using the elephant trunk technique. Ann Vasc Surg. 2000; $14: 612-9$

11. Safi HJ, Miller CC III, Estrera AL, Huynh TT, Rubenstein FS, Subramaniam MH, et al. Staged repair of extensive aortic aneurysms: morbidity and mortality in the elephant trunk technique. Circulation. 2001;104:2938-42.

12. LeMaire SA, Carter SA, Coselli JS. The elephant trunk technique for staged repair of complex aneurysms of the entire thoracic aorta. Ann Thorac Surg. 2006;81: 1561-9.

13. Kouchoukos NT, Mauney MC, Masetti P, Castner CF. Single-stage repair of extensive thoracic aortic aneurysms: experience with the arch-first technique and bilateral anterior thoracotomy. J Thorac Cardiovasc Surg. 2004;128: 669-76.

14. Kazui T, Tamiya Y, Tanaka T, Komatsu S. Extended aortic replacement for acute type A dissection with the tear in the descending aorta. J Thorac Cardiovasc Surg. 1996;112:973-8.

15. Mizuno T, Toyama M, Tabuchi N, Wu H, Sunamori M. Stented elephant trunk procedure combined with ascending aorta and arch replacement for acute type A aortic dissection. Eur J Cardiothorac Surg. 2002;22:504-9.

16. Flores J, Kunihara T, Shiiya N, Yoshimoto K, Matsuzaki K, Yasuda K. Extensive deployment of the stented elephant trunk is associated with an increased risk of spinal cord injury. J Thorac Cardiovasc Surg. 2006;131:336-42. 